\title{
Effectiveness of the Self-Care Sandbag Exercise Program for the Osteoarthritis of Knee Patients in the Secondary Care in Thailand: Randomized Control Trial
}

\author{
Tanthanapanyakorn, P., ${ }^{1 *}$ Sanguanchue, A., ${ }^{1}$ Khuntikulanon, N., ${ }^{1}$ Chanmalee, S. ${ }^{1}$, \\ Cetthakrikul, S. ${ }^{2}$ and Wongpituk, $\mathbf{K}^{1}{ }^{1}$
}

${ }^{1}$ Department of Public Health, Faculty of Public Health, Valaya Alongkorn Rajabhat University under the Royal Patronage, Thailand, E-mail: phannathat.tan@vru.ac.th, aree.sa@vru.ac.th,nonlapan.khun@vru.ac.th, sasiwimol.chan@vru.ac.th, klarmsu@gmail.com

${ }^{2}$ Department of Physical Therapy, School of Allied Health Sciences, Walailak University, Thailand

E-mail: scetthakrikul@gmail.com

*Corresponding Author

DOI: https://doi.org/10.52939/ijg.v17i5.2051

\begin{abstract}
Osteoarthritis is the most common form of arthritis in the knee. It is occurring when the protective cartilage that cushions the ends of your bones wears down over time and mostly often in people 50 years of age and older. The study was designed to randomized control trial and aimed to compare before and after the 3-months intervention program on self-care sandbag exercise program for knee osteoarthritis patients in the secondary care in Thailand. They were divided into 2 groups, the intervention group, and the control group by using the probability sampling. Seventy knee osteoarthritis patients participated in this study. Both of group were assessed the Oxford knee score and the knowledge of knee osteoarthritis at the baseline and after 3-months intervention program. The Independent t-test and Paired sample t-test were used for comparison the all parameters between and within the group, respectively. The results showed that the Oxford pain score of knee osteoarthritis was decreased scientifically more than the control group $(p<0.001)$ and the knowledge of knee osteoarthritis was increased significantly $(p<0.001)$. In conclusion, the self-care sandbag exercise program can reduce the severity of pain among knee osteoarthritis patients and can promote to the usaul care for the osteoarthritis pateintes.
\end{abstract}

\section{Introduction}

Osteoarthritis $(\mathrm{OA})$ of the knee is the most common type of arthritis and the major cause of chronic musculoskeletal pain and mobility disability in the elderly (Gay et al., 2016). It is being encountered with increasing frequency as the population continues to age, and therefore represents a significant burden on healthcare provision ( Zampogna et al., 2020). Risk factors of OA of the knee include older age, female sex, obesity, osteoporosis, occupation, sports activities, previous trauma, muscle weakness or dysfunction, proprioceptive deficit and genetic factors (Taglietti et al., 2018).

So, the Osteoarthritis of the knee was absolutely known as wear-and-tear arthritis. It is a condition in which the natural cushioning between joints cartilage wears away ( Jevsevar, 2013). When this occur, the bones of the joints rub more closely against one another with less of the shock-absorbing benefits of cartilage.

The Symptoms of knee osteoarthritis may include: pain that increases when you are active, but gets a little better with rest, swelling, feeling of warmth in the joint, stiffness in the knee, especially in the morning or when you have been sitting for a while and decrease in mobility of the knee, making it difficult to get in and out of chairs or cars, use the stairs, or walk (Chopra, 2013). A recent estimation revealed that approximately 250 million individuals are suffering from OA worldwide, and the knee joint is the most frequently affected joint with an incidence of 16$17 \%$ among people aged 50 to 75 years (Huang et al., 2017) . Chronic pain and impaired physical function are recognized as the main issues affecting quality of life for patients with $\mathrm{OA}$ of the knee (Nelson et al, 2014) and guides for improving the long-term outcomes of pain and physical function for patients with OA of the knee (Jorgensen et al., 2017).

As mentioned above, osteoarthritis of knee has changed for the worse in adults and elderly. It is a major problem in the health system of the Thailand. The National survey reported that, OA of the knee is 
the most common diseases that may cause to disability in the elderly (McAlindon et al., 2014) and effect to the quality of life of the patients and family. This finding consistent with the report of Thai elderly health status in the year 2013. The result showed that prevalence of knee osteoarthritis in the elderly was at $12.3 \%$ (Fransen et al., 2015). Non-pharmacological treatment and complementary therapy are effectively reducing the worse symptom or complication ( Peeler et al., 2015) . The selfmanagement exercise is the most intervention to decrease the severity, especially the knee osteoarthritis on early and middle stages. They should be given an exercise program on their own and improve the knowledge about knee osteoarthritis from the secondary hospital or primary hospital ( Rerkrai, 2016) . Conclusion, Self-exercise of the knee can effectively slow down the severity of the disease and can retard the disease progression to the severe stage (Narin et al., 2015).

From the previous interventions, they were used the regular exercise including aerobic dance, slow walking and other for maintaining the knee and leg exercise movement but prevalence of the OA of the knee was increased in every year, especially in central region is still high. In addition, another intervention did not proper for reduce the knee pain severity. This intervention program is the comprehensive intervention by adapting the sandbag for promoting the stretching and strengthening of the muscle and knee joint. Exercise is one of the effective methods that is commonly prescribed for patients with knee OA to alleviate pain and improve physical function (Anwer and Alghadir, 2014).

Conclusion, this intervention was integrated sandbag with the exercise program which it can reduce the severity of knee osteoarthritis. Using the sandbag can improve the muscle stretching and muscle strengthening on leg and knee of knee osteoarthritis. This intervention program was based on the information-motivation-behavioral skill model to develop the model for raising the knee exercise management ( Fisher et al., 2003). This study was aimed to evaluate the effectiveness of self-care sandbag exercise program intervention program to reduce knee osteoarthritis symptoms and improve the physical functioning of knee osteoarthritis patients in the secondary hospital in Thailand.

\section{Methodology}

This is a randomized control trial to appraise before and after the 3-months intervention program on selfcare sandbag exercise program for knee osteoarthritis patients in the secondary care of Thailand. This study was carried out in the secondary care of central region, Thailand. This is a suburban area in Thailand with several characteristics of the population. It was separated into 2 groups by simple random sampling: the intervention group who were received the self-care sandbag exercise program and the control group who were received the health education booklets and usual care. The data collection was conducted between June 2019 to March 2020 through an interview and assessment forms. This program was designed to 6-weeks exercise program and used the 3-months to follow-up and this study did not blind.

\subsection{Participants}

The participants were recruited from the secondary care in Thailand with high prevalence of knee osteoarthritis patients (Peeler et al., 2015) who were registered in the medical care at least 1 year and aged range from 45 years and older. Moreover, the participants who were measured the Oxford knee score to recruite the participants in to the study. This study was selected the participants with the level 1 to 3 of knee osteoarthritis. The participants were participated in this study with the consent from and singed the name. The simple random sampling was used to recruit the respondents as per the inclusion and separated into 2 groups. The exclusion criteria consisted of 1.) the participants had been diagnosed as having the mental health problem such as depression, stress, anxiety 2.) the participants who were received another self-exercise program in as the same time with study or the previous 3 -months. In addition, they were excluded who had physical problems that limited to exercise.

The most common cause of osteoarthritis of the knee is age. Almost everyone will eventually develop some degree of knee osteoarthritis. However, several factors increase the risk of developing significant arthritis at an earlier age. So, the knee osteoarthritis was raised to the crucial situation in Thailand. Based on the simple random sampling was carried out in the secondary care hospital. Based on simple random sampling, the participants from the secondary hostipal of Thailand were placed to intervention and control groups. At beginning, the total 50 of participants from each group were recruited to this study by voluntary.

After the baseline measurement, total 80 participants who met the criteria were selected to this study consisted of 40 participants in the intervention group and 40 participants in the control group. After the 3-months interventions program, 35 participants from the intervention group and 35 participants from the control group who were finished the program. Thus, the total 70 participants were performed post-test measurement. 
As the sample size of this study was calculated by the G-power program which using the confidence interval was $95 \%$ study, allowable error was $5 \%$ and effect size was 0.45 (Juhl et al., 2014). After calculation the sample size by the G-power program, the total sample size was used 80 participants.

\subsection{Material and Procedure}

Knee osteoarthritis is the most common form of arthritis in the knee. It is occurring when the protective cartilage that cushions the ends of bones wears down over time and mostly often in people 50 years of age and older (Roos and Arden, 2015). The knee osteoarthritis patients were approached by the physician and who were registered on the medical treatment systems from the secondary care unit in Thailand. At the beginning, the participants were assessed the level of knee osteoarthritis by the physician along with reviewed the medical record until 1 year before. The patients who had level IV of knee osteoarthritis were excluded from this study that limited for exercise. The total number of 70 participants aged 50 years old and older and diagnosed with the knee osteoarthritis level I to II were included to the study by voluntary. They did not have mobility restrictions or medical contraindications including no history of knee injuries and no restrictions or medical conditions.

The self-care sandbag exercise program was integrated the $1 \mathrm{~kg}$ of sandbag for weighting exercises in the knee joint followed by the intervention designed. The intervention was implemented and monitored by the researcher team consisted of the physician, nurse, physical therapy staff and public health staff along with the intervention plan. Moreover, this intervention was considered by the medical and physical therapy and edit to proper followed by the supervisor suggestion. This intervention was adapted the informationmotivation-behavioral skill to conduct the intervention detail (Fisher et al., 2003). The intervention was conducted to 6-weeks at the secondary care unit by using 4 hours per time.

The first week, the researcher was explained the natural of knee osteoarthritis disease, self-care of patients with osteoarthritis prevention, brief performing exercises on the knee osteoarthritis to delay the osteoarthritis by using the picture chart, slide presentation and VDO media. These materials can be easily understood by the patient. Moreover, the researcher team was informed about the study protocol, the benefit and risky when the participants joined with the intervention program. The second week, the researcher was aimed to raise awareness for practicing the self-care exercise by using the sandbag. This section was given the knowledge of performing knee exercises for knee osteoarthritis patients and assigned $1 \mathrm{~kg}$ sandbags to each patient. At the beginning, the researcher team was demonstrated the practice of knee exercises by using sandbag to slowly and assigned for the patients to follow the step by step. So, the participant was received the educational booklet of self-care sandbag exercise which they can implement the exercise at their house followed by the step postures. For third to five weeks, the researcher was aimed to make an appointment to follow-up with knee pain and complications. As well as, the researcher was purposed to review the self-care of knee exercises with one by one as specified. Moreover, the group discussion was established for sharing the experiences with each other and brought to develop the appropriate of sandbag exercise step. The last week, the researcher was measured the all of parameters and promoted to practice the sandbag exercise at their house at least 3 times/week and appointment to meet after 3-months for measuring the outcome and conclusion the result after the participant who were finished the program. The control group were received the usual care and health education booklet who were measured the same parameter with the intervention group.

The self-care sandbag exercise program consisted of 3 steps: step 1 was the stretching or warm-up for body. This step has 3 position consisted of straight sit with the legs extended, sit upright and kick the feet floating on the floor and straight sit with the back against the chair by using 10 set per leg side (Figure 1). Step 2 was designed to use the sandbag with the exercise posture. The postures consisted of straight sit with the legs extended for increasing the muscle strength, sleep on the stomach, sleep on the back and sleep on the side by using 10 set per leg side (Figure 2). Step 3 was stretched without the sandbag for cool-down consisted of lie on the back and support by using pillow, lie on the back and raise the legs, lie on the back and align the buttocks and straight sit with the back against the chair by using 10 set per leg side. So, every posture was recommended to practice with 2 rounds per position on 10 set per rounds (Figure 3). The intervention group was scheduled to perform self-care sandbag exercise with 3 days/week and every week, the researcher was appointed to meet for following the skill and pain level of knee osteoarthritis on week 2-5 of intervention program. The exercise program was considered by social distancing and prevent to infect the Covid pandemic and distribution to the each other. The control group did the leisure exercise activity by themselves and received the health education. The study area was 
placed at the secondary health care unit in the central region of Thailand and the control group was performed the exercise at their own place. The trained co-researchers were measured, recorded and collected all tool and all parameters at the baseline and 3-months intervention program.
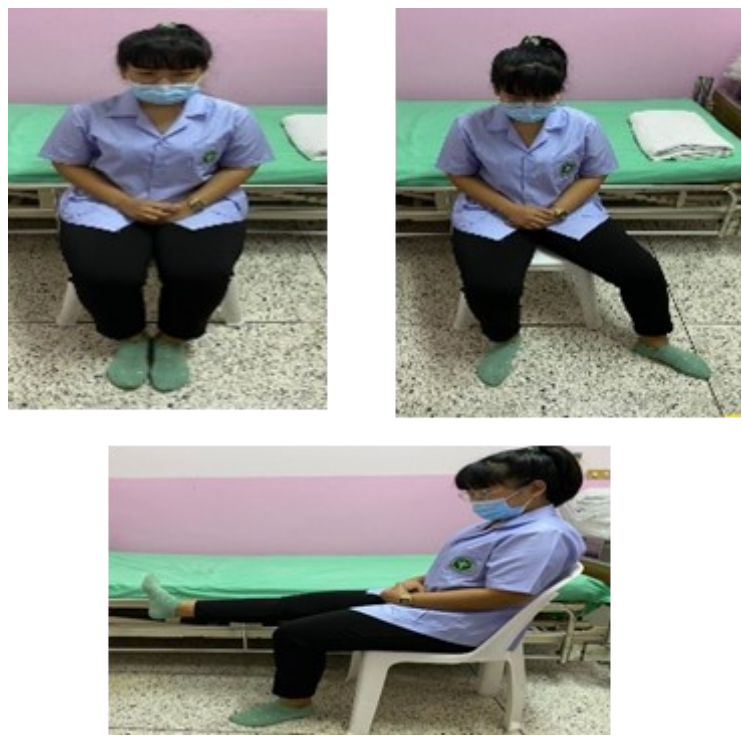

Figure 1: Self-care sandbag exercise program on warm-up stage. This stage was helped relax of muscle and ready to exercise
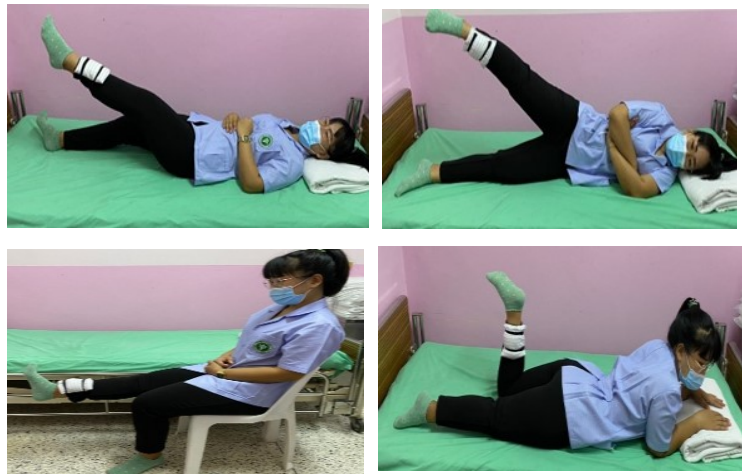

Figure 2: Self-care sandbag exercise program on exercise stage. This stage was interggeted the sandbag to exercises for reducing pain and increase muscle strengthening
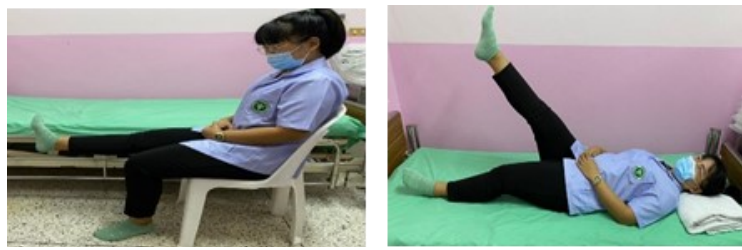

Figure 3: Self-care sandbag exercise program on cool down stage. This satge was helped the functioning of the heart, blood vessels, and muscles return to normal and makes no muscleaches after exercise

\subsection{Instruments}

The self-care sandbag exercise program was designed to implement in this study. The instrument was used the paper recorded form and the structural interview form by face to face interview with the social distancing for preventing the Coronavirus-19 distribution and the mask was used along with the interview period. The detail of the instrument can describe as follow:

Part 1: The Socio-demographic data questionnaire: The total of this part was 7 questions. This part was to record socio-demographic data such as age, gender, educational level, occupational, income sufficiency, and caregiver.

Part 2: The Oxford knee score (OKS): OKS is a 12item patient-reported and developed to assess function and pain after total knee replacement (TKR) or surgery (arthroplasty). It is short, reproducible, valid and sensitive to clinically important changes. The OKS was designed by Dawson et al., (1998) and validated to measure pain and function after total knee replacement. The OKS was originally developed and validated for use with individuals undergoing knee arthroplasty but has also been used to measure outcomes in pharmacological treatments, after osteotomies, following rehabilitation or with fractures (Harris et al., 2016). The OKS is a patient reported outcome measure that consists of 12 questions about an individual's activities of daily living and how they have been affected by pain over the preceding four weeks. The OKS was originally developed, it was designed to be as simple as possible in order to promote use. The original scoring system was a 1-5 where one represented the best outcome confusing in practice and adaptations began to appear so the original authors developed a new scoring system from 0-4 where four is the best outcome and total scores range from 0 (worst outcome) to 48 (best outcome) (Harris et al., 2016).

The OKS has demonstrated strong test-retest reliability in its original testing (Harris et a., 2016). A 2016 systematic review of 23 studies found good evidence of its reproducibility (Beard et al., 2015). Moreover, the validity showed that the OKS was correlated significantly with similar measures (American Knee Society Score, SF-36particularly the pain and physical function domains). 
Part 3: The knowledge of knee osteoarthritis assessment form; The total of this part was to 10 questions. Each question has 4 answers choice. The score ranged from 0-10 points. The high total scores indicated good level of knowledge. The sandbag exercise program Retro Thai dancing exercise program was measured the validity by peer from 3 experts on physical therapy, medical and public health provider and reported the IOC value. The IOC value was more than 0.5 . The reliability was tested in 30 knee osteoarthritis which it is locate at the central region of Thailand same as the study area whose characteristics were similar to those of the participants. The reliability of Cronbach's alpha coefficients was more than 0.90 , it was the acceptable of the measurement tool.

\subsection{Ethical Consideration}

This study was approved by the ethics review committee for health research. Researcher and coresearchers were told the participants about the study protocol and risk of self-care sandbag exercise before they signed a consent form.

\subsection{Statistical Analysis}

The data analysis was performed using SPSS 22.0 for windows. 1.) The socio-demographic data were analyzed by descriptive statistic such as number, percentage, mean, standard divion and range. The chi-square test and Pearson's correlation coefficients were used to estimate baseline characteristics between group. The KolmogorovSimirnov Goodness of Fit test was used to measure the normality test and normal distribution. As the results showed that there was the normal distribution in all variable. The Independent t-test was used to compare before and after the 3 -months intervention program between the group and the aired sample t-test was used to compare before and after 3-months intervention program within the group. All results with $\mathrm{p}<0.05$ was considered statistically significant.

\section{Result}

A total of 80 participants had 5 dropouts from intervention group and 5 dropouts from control group during the 3-monthhs intervention program. During the last week of the program, some participants could not join the self-care sandbag exercise because they had worse symptom that effect to their exercise. So, there were 70 participants (intervention group; $\mathrm{n}=35$ and control group; $\mathrm{n}=35$ ) in the total who participated in this study.

The socio-demographic and economic status and outcome variables were similar between both groups showed in Table 1 . The results showed a total of 70 participants were $70.2 \pm 8.8$ years old with an average of weight was $65.1 \pm 2.3$ kilograms. The participants were male more than female $(64.2 \%$ and $35.8 \%$, respectively). Most of them had a secondary school (35.4\%), 83.1\% were married and $70.8 \%$ were merchanted or employed. Their monthly were sufficiency of income) $87.1 \%$ ). The majority of the samples who haven't caregiver accounted for $51.4 \%$. Moreover, the majority of the participants who were had one side of knee disformed (72.3\%) and $71.4 \%$ of them had less than 1 year with knee osteoarthritis They were visited to the professional sectors more than folk sector $(51.4 \%)$ and $61.4 \%$ had universal coverage.

At the baseline, the mean change of all parameters (mean \pm SD.) between the intervention group and control group were not difference significantly $(\mathrm{p}>0.05)$. The mean change of all the parameters (mean $\pm \mathrm{SD}$.) within group and between group after the 3-months intervention program were compared. The results revelated that Oxford knee score and the knowledge about knee osteoarthritis in the intervention group was significantly increased more than the control group $(p<0.001)$. The comparison of all parameters within the intervention group at the baseline and 3-months intervention program found that the Oxford knee score was at $23.03 \pm 1.56$ and increased significantly to $30.45 \pm 1.32(\mathrm{p}<0.001)$. The knowledge about knee osteoarthritis was at $7.02 \pm 0.78$ and increased significantly to $9.66 \pm 0.48(p<0.001)$. In addition, the comparison of all parameters within the intervention group at the baseline and 3-months intervention program found that the Oxford knee score was at $21.34 \pm 1.48$ and increased significantly to $27.66 \pm 1.91 \quad(\mathrm{p}<0.001)$. The knowledge about knee osteoarthritis was at $6.69 \pm 0.97$ and increased significantly to $8.09 \pm 0.89(\mathrm{p}<0.001)$ (Table 1 and Table 2).

\section{Discussion}

The study design was experimental and controlled, which suitable for measuring the effectiveness of intervention program (Oiestad et al., 2013). The baseline value of all variable and outcome was no difference between 2 groups. 
Table 1: The baseline characteristics and outcome variable at the baseline between intervention and control, $\mathrm{p}$-value $=0.05$

\begin{tabular}{|c|c|c|c|c|}
\hline Variables & Total & Intervention group & Control group & p-value \\
\hline \multicolumn{5}{|l|}{ Age (Years) } \\
\hline $\begin{array}{l}\text { Mean } \pm \text { SD. } \\
\text { Weight (Kg) }\end{array}$ & $70.2 \pm 8.8$ & $69.02 \pm 6.7$ & $72.11 \pm 5.9$ & 0.124 \\
\hline Mean \pm SD. & $65.1 \pm 2.3$ & $69.3 \pm 8.6$ & $64.3 \pm 6.3$ & 0.124 \\
\hline Male & $45(64.2 \%)$ & $22(62.8 \%)$ & $23(65.7 \%)$ & 0.921 \\
\hline Female & $25(35.8 \%)$ & $13(38.2 \%)$ & $12(34.3 \%)$ & \\
\hline \multicolumn{5}{|l|}{ Marital Status } \\
\hline Married & $54(83.1 \%)$ & $27(84.4 \%)$ & $27(81.8 \%)$ & 0.788 \\
\hline Single & $11(16.9 \%)$ & $30(15.63 \%)$ & $6(18.2 \%)$ & \\
\hline \multicolumn{5}{|l|}{ Education Level } \\
\hline Primary School & $14(21.5 \%)$ & $7(21.9 \%)$ & $7(21.2 \%)$ & 0.922 \\
\hline Secondary School & $23(35.4 \%)$ & $11(34.4 \%)$ & $12(36.4 \%)$ & \\
\hline Diploma & $13(20.0 \%)$ & $6(18.8 \%)$ & $7(21.2 \%)$ & \\
\hline Bachelor degree & $15(23.1 \%)$ & $8(25.0 \%)$ & $7(21.2 \%)$ & \\
\hline \multicolumn{5}{|l|}{ Occupational } \\
\hline Merchants/Employee & $46(70.8 \%)$ & $24(75.0 \%)$ & $22(66.6 \%)$ & 0.801 \\
\hline Agriculturist & $15(23.1 \%)$ & $6(18.9 \%)$ & $9(27.3 \%)$ & \\
\hline Government Officer & $4(6.1 \%)$ & $2(6.1 \%)$ & $2(6.1 \%)$ & \\
\hline \multicolumn{5}{|l|}{ Income Sufficiency } \\
\hline Sufficiency & $61(87.1 \%)$ & $31(88.6 \%)$ & $30(85.7 \%)$ & 0.457 \\
\hline Insufficiency & $9(12.8 \%)$ & $4(11.4 \%)$ & $5(14.3 \%)$ & \\
\hline \multicolumn{5}{|l|}{ Caregiver } \\
\hline Yes & $34(48.6 \%)$ & $18(51.4 \%)$ & $16(45.7 \%)$ & 0.120 \\
\hline No & $36(51.4 \%)$ & $17(48.6 \%)$ & $19(54.3 \%)$ & \\
\hline \multicolumn{5}{|c|}{ Number of knees disformed } \\
\hline One side & $52(72.3 \%)$ & $24(68.6 \%)$ & $28(80.0 \%)$ & 0.321 \\
\hline Two side & $18(25.7 \%)$ & $11(31.4 \%)$ & $7(20.0 \%)$ & \\
\hline \multicolumn{5}{|l|}{ Time of OA knee } \\
\hline$<1$ year & $50(71.4 \%)$ & $18(51.4 \%)$ & $22(68.8 \%)$ & 0.145 \\
\hline$>1$ year and equal & $20(29.6 \%)$ & $17(48.6 \%)$ & $13(31.2 \%)$ & \\
\hline \multicolumn{5}{|l|}{ Treatment options } \\
\hline Folk sector & $34(48.6 \%)$ & $18(51.4 \%)$ & $16(45.7 \%)$ & 0.222 \\
\hline Professional sector & $36(51.4 \%)$ & $17(48.6 \%)$ & $19(54.3 \%)$ & \\
\hline \multicolumn{5}{|l|}{ Health insurance } \\
\hline Universal coverage & $43(61.4 \%)$ & $23(65.7 \%)$ & $20(51.7 \%)$ & 0.143 \\
\hline Government welfare & $27(38.6 \%)$ & $12(34.3 \%)$ & $15(42.8 \%)$ & \\
\hline
\end{tabular}

REMARK: Data were analyzed with Chi-square test and independent t-test. *Statistically significant level at the 0.05 .

The intervention groups showed the Oxford knee score and knowledge about $\mathrm{OA}$ of the knee were increased significantly between 2 groups after the 3months intervention program $(\mathrm{p}<0.001)$. The result is consistent with the study of Hongbo Chen et al., (2013) whose study the effects of a home-based exercise intervention on elderly patients with knee osteoarthritis. This study was aimed to evaluate the effectiveness of intervention program to reduce knee osteoarthritis symptoms and improve the physical functioning of elderly patients. The finding showed that at week 12, the pretest-posttest changes was decreased in pain intensity and stiffness, with the intervention group exhibiting significantly larger improvements on both measures than the control group. 
Table 2: Mean difference of all parameters between intervention group and control group

\begin{tabular}{lccc}
\hline \multicolumn{1}{c}{ Variable } & $\begin{array}{c}\text { Baseline } \\
\text { (Mean } \pm \text { SD.) }\end{array}$ & $\begin{array}{c}\text { 3-months Intervention } \\
\text { Mean } \pm \text { SD.) }\end{array}$ & p-value (a) \\
\hline Oxford knee score & & & \\
Intervention group & $23.03 \pm 1.56$ & $30.45 \pm 1.32$ & $<\mathbf{0 . 0 0 1 *}$ \\
Control group & $21.34 \pm 1.48$ & $27.66 \pm 1.91$ & $<\mathbf{0 . 0 0 1 *}$ \\
p-value (b) & 0.873 & $<\mathbf{0 . 0 0 1 *}$ & \\
Knowledge of osteoarthritis & & & \\
Intervention group & $7.02 \pm 0.78$ & $9.66 \pm 0.48$ & $<\mathbf{0 . 0 0 1 *}^{*}$ \\
Control group & $6.69 \pm 0.97$ & $8.09 \pm 0.89$ & $<\mathbf{0 . 0 0 1 *}$ \\
p-value (b) & 0.333 & $<\mathbf{0 . 0 0 1}$ & \\
\hline
\end{tabular}

REMARK: Data were analyzed with paired sample t-test within group ( $p$-value (a)) and independent t-test between group (p-value (b)). *Statistically significant at the 0.05 level ( $p$-value $<0.05)$

Similarity, Shahnawaz et al., (2016) whose study the effect of home exercise program in patients with knee Osteoarthritis. The result showed that significant improvements in pain and function in individuals with knee OA. So, home exercise programs with and without supervised clinic-based exercises was effectively helped in the rehabilitation of knee OA. In addition, the finding consisted with Supada et al., (2020) whose study the effects of exercise self-management program on disease severity among adults with knee osteoarthritis. This research findings revealed that after the participants were participated in the program. The sample who were showed a statistically significant lower level of disease severity $(\mathrm{p}<0.05)$. The effect of the program can enhance adults with knee osteoarthritis skills and techniques in regular. And similarity with Yaser and Razie (2020) whose studies the effect of a water jogging exercise course on older men with knee osteoarthritis. This objective was aimed to investigate the effect of a water jogging training program on the symptoms of knee osteoarthritis in elderly. The result showed that, between pre-posttest was different significantly with mean of symptoms, knee pain, motor function in daily activities $(\mathrm{p}<0.05)$.

This finding might explain that; firstly, knee osteoarthritis is a major public health problem which is more common in adults aged 50 years and over which consisted with the inclusion criteria of this study. The risk of developing the disease and the degree of severity increases with age, and it can affect physical health, mental health, and quality of life. The mainstays of care are to prevent complications and to reduce disease severity. It is necessary for adults with knee osteoarthritis to follow proper self-management, to exercise regularly (Wang et al., 2016) and to reduce the disease severity. So, appropriate exercise selfmanagement was integrated with the sandbag and movement posture will delay disease progression and reduce disease severity among adults with knee osteoarthritis. Secondly, this program was designed on 6-weeks intervention and used 3 months followup. The self-care exercise program was adapted the sandbag to administrate the knee join with 3 step of intervention design. The participants in experimental group was performed the self-care sandbag exercise for 30 minutes per day, 3 days per week, for 6 consecutive weeks. The program was followed the standard of physical activity from World Health Organization (2004) mentioned that everyone should had the moderate-intensity exercise or taken time on 150 minutes/week because, this can help to prevent the chronic diseases, promote the work of the body system, stimulate the blood circulation and reduce the body composition. Thus, the control group was continued their normal daily activity with the health educational booklet. From the coronavirus pandemic, the social distancing and mask prevention should be concerned to conduct the intervention program. Thirdly, the participants from intervention group had the scored of oxford knee score more than control group. The overweight adults who were received the intervention exercise program had more reduced the pain symptom and improved the self-knowledge which related to the health behavior changed. This finding is consistent with the behavior theory of Bandura (1986) said that the individual behavior is not caused by only reinforcement and punishment by external factor, but the person can do to control their thinking, feeling and acting by using 3-step of self-regular process includes:

1.) Self-observation; the experimental group was given information on their own pain score and knowledge about knee osteoarthritis. After that, the participants were observed their exercise behavior and found that they had relief the pain symptom through sandbag exercise followed by the intervention program. This may be caused that the participants have awareness of self-exercise by 
setting their goal to maintain on 30 minutes per day, 3 days per week, for 6 consecutive weeks.

2.) Judgment process; from the evaluation of the self-exercise behavior that was successfully compared to their goals and member. This may be caused the participants to realize the value of selfexercise. Thus, they can decide to plan the selfexercise by using sandbag and remind themselves by recording their self-exercise behavior. It makes the sample can clear and continue to exercise until 6 weeks and sustain to exercise for their live.

3.) Self-reaction; it is an incentive or reinforcement process in the experimental group to achieve continually self-exercise behavior by recoding self-appreciation, expressing the feelings through social media, reward their belongings when able to reduce pain severity. The participants can maintain the self-exercise with sandbag and affected to lose weight, they will be the great role models for people who have not been able to exercise successfully.

The sandbag exercise program is the instrumental to reduce the pain symptom, increase physical activity and takes pride in enhancing their exercise performance. Thus, the compliment was encouraged to exercise and help for promoting the sustainable exercise behavior.

\section{Conclusion}

The self-care sandbag exercise program was designed as the individual exercise program, was easy and simply to perform this program by themselves with the appropriate positions for the knee osteoarthritis patients. The results showed that the intervention program can increase the Oxford knee score and reported they had the best outcome of knee osteoarthritis pain and impeoved the knowledge on osteoarthritis. This program implementation, the result can be used for developing the campaign by using self-care sandbag exercise program to reduce the pain severity and prevent the complications from the diseases. Moreover the results of this study are promising which can be benefited in patients' management of osteoarthritis especially those with mild symptom. This finding indicated that a public health provider could integrate the self-care sandbag exercise program with usual care to promote the good quality of life in knee osteoarthritis pateints. There were 2 limitations in this study. Firstly, the study area is the secondary hospital.

Thus, the results cannot generalize to the primary hospital or tertiary hospital duo to differences in disease complication, lifestyles, socio-demographic and economic status. Secondly, This study had limitation in terms of the duration such as short duration of the exercise program which participants were received 6-weeks of self-care sandbag exercise sessions by the physical therapist and used a 3month follow-up all of the outcomes. The future study should apply this program to longer follow-up time after finishing the program to determine sustain ability of the program. The study were focused on pain score and knowledge. It would be benefit to add in additional parameters such as reduce in the intake of pain killer and improvement in the quality of life. The future study should use the GIS to analyze knee osteoarthritis trends, analyze access to public health services, analyze the built environment for knee osteoarthritis pateintes, and design community health communications programs. The tremendous potential of GIS to benefit the health care services. Both public and private sectors are developing innovative ways to harness the data integration and spatial visualization power of GIS to health care systems. Futuremore, the future study should focus or describe on spatial distribution or analysis by considering location as a parameter for understanding exercise characteristics. The stronger

\section{Acknowledgement}

The authors are grateful to all participants for their willing participation in this study. We would like to give a special thanks to the director of secondary health care hospital and head, department of orthopedic for the generosity location to conduct the self-care sandbag exercise program.

\section{References}

Anwer, S. and Alghadir, A., 2014, Effect of Isometric Quadriceps Exercise on Muscle Strength, Pain, and Function in Patients with Knee Osteoarthritis: A Randomized Controlled Study. Journal of Physical Therapy Science, Vol. 26, 5, 745-748.

Bandura, A., 1986, Social Foundation of Though and Action: A social Cognitive Theory. Migigan: Prentice-Hall.

Beard, D., Harris, K., Dawson, J., Doll, H., Murray, D. W., Carr, A. J. and Price, A. J., 2015, Meaningful Changes for the Oxford Hip and Knee Scores After Joint Replacement Surgery. $J$ Clin Epidemiol., Vol. 68(1):73-79.

Chen, H., Zheng, X., Huang, H., Liu, C., Wan, Q. and Shang., S., 2019, The Effects of a HomeBased Exercise Intervention on Elderly Patients with Knee Osteoarthritis: A Quasi-Experimental Study. BMC Musculoskelet Disord, Vol. 20(160), 1-11. 
Chopra, A., 2013, The COPCORD World of Musculoskeletal Pain and Arthritis. Rheumatology, Vol. 52, 1925-1928.

Dawson, J., Fitzpatrick, R., Murray, D. and Carr, A., 1998, Questionnaire on the Perceptions of Patients about Total Knee Replacement. $J$ Bone Joint Surg Br, Vol. 80, 63-69.

Fransen, M., McConnell, S., Harmer, A. R., Van der Esch, M., Simic, M. and Bennell, K. L., 2015, Exercise for Osteoarthritis of the Knee. Cochrane Database of Systematic Reviews, Vol. 49(24). 1-6.

Fisher, W. A., Fisher, J. D. and Harman, J., 2003, The Information-Motivation-Behavioral Skill Model: A General Social Psychological Approach to Understanding Promoting Health Behavior, Social psychological foundation of Health and Illness, Blackwell, Malden, MA, 82106.

Gay, C., Chabaud, A., Guilley, E. and Coudeyre, E., 2016, Educating Patients about the Benefits of Physical Activity and Exercise for their Hip and Knee Osteoarthritis. Systematic literature review. Annals of Physical and Rehabilitation Medicine, Vol. 59, 3, 174-183.

Harris, K., Dawson, J., Gibbons, E., Lim, C., Beard, D., Fitzpatrick, R. and Price, A., 2016, Systematic Review of Measurement Properties of Patient-Reported Outcome Measures Used in Patients Undergoing Hip And Knee Arthroplasty. Patient Relat Outcome Meas, Vol. 7, 101-108.

Huang, K. H., Hsieh, R. L. and Lee, W. C., 2017, Pain, Physical Function, and Health in Patients with Knee Osteoarthritis. Rehabil Nurse, Vol. 42, 235-241.

Jevsevar, D., 2013, The Treatment of Osteoarthritis of the Knee - 2nd Edition: Clinical Practice Guideline Work Group. Journal of the American Academy of Orthopaedic Surgeon, Vol. 21(9), 571-576.

Jorgensen, P. B., Bogh, S. B., Kierkegaard, S., Sorensen, H., Odgaard, A., Soballe, K., Mechlenburg, I., 2017, The Efficacy of Early Initiated, Supervised, Progressive Resistance Training Compared to Unsupervised, HomeBased Exercise After Unicompartmental Knee Arthroplasty: a Single-Blinded Randomized Controlled Trial. Clin Rehabil, Vol. 31, 61-70.

Juhl, C., Christensen, R., Roos, E. M., Zhang, W. and Lund, H., 2014, Impact of Exercise Type and Dose on Pain and Disability in Knee Osteoarthritis: A Systematic Review and MetaRegression Analysis of Randomized Controlled Trials, Arthritis Rheumatol, Vol. 66, 622-636.
McAlindon, T. E., Bannuru, R. R., Sullivan, M., Arden, N., Berenbaum, F., Bierma-Zeinstra, S. and Kawaguchi, H., 2014, OARSI Guidelines for the Non-Surgical Management of Knee Osteoarthritis. Osteoarthritis and Cartilage, Vol. 22, 3, 363-388.

Narin, R., Taunrat, W. and Booncheang, W., 2015, Development of a Community Participation Program for Caring Older Adults With Knee Osteoarthritis. Nursing Journal, Vol. 42(3), 170181.

Nelson, A. E., Allen, K. D., Golightly, Y. M., Goode, A. P. and Jordan, J. M, 2014, A Systematic Review of Recommendations and Guidelines for the Management of Osteoarthritis: the Chronic Osteoarthritis Management Initiative of the U.S. Bone And Joint Initiative. Semin Arthritis Rheum, Vol. 43, 701-712.

Oiestad, B. E, Osteras, N., Frobell, R., Grotle, M., Brogger, H. and Risberg, M. A., 2013, Efficacy of Strength and Aerobic Exercise on PatientReported Outcomes and Structural Changes in Patients with Knee Osteoarthritis: Study Protocol for a Randomized Controlled Trial. BMC Musculoskelet Disord, Vol. 14, 1-10.

Peeler, J., Christian, M., Cooper, J., Leiter, J. and MacDonald, P., 2015, Managing Knee Osteoarthritis: the Effects of Body Weight Supported Physical Activity on Joint Pain, Function, and thigh Muscle Strength. Clin J Sport Med, Vol. 25, 518-523.

Rerkrai, C., 2016, The Effect of Self-Management Focusing on Exercise Program on Disease Severity Among Adult Patients with Knee Osteoarthritis. Bangkok, Chulalongkorn University. 10.14457/CU.the.2016.613.

Roos, E. M. and Arden, N. K., 2015, Strategies for the Prevention of Knee Osteoarthritis. Nature Review Rheumatoloty, Vol. 12(2), 92-101.

Shahnawaz, A., Ahmad, A. and Jean, M. B., 2016, Effect of Home Exercise Program in Patients with Knee Osteoarthritis: A Systematic Review and Meta-analysis. Journal of GERIATRIC Physical Therapy, Vol. 39(1), 38-48.

Supada, B., Rangsiya, N. and Siwaporn, A., 2020, Effects of Exercise Self-Management Program on Disease Severity Among Adults with Knee Osteoarthritis. Nursing Journal, Vol. 47, 300313.

Taglietti, M., Facci, L. M., Trelha, C. S., de Melo, F. C., da Silva, D. W., Sawczuk, G. and Cardoso, J. R., 2018 , Effectiveness of Aquatic Exercises Compared to Patient Education on Health Status in Individuals with Knee 
Osteoarthritis: A Randomized Controlled Trial. Clinical rehabilitation, Vol. 32(6), 766-776.

Wang, P., Yang, L., Liu, C., Wei, X., Yang, X. and Zhou, Y., 2016, Effects of Whole-Body Vibration Exercise Associated with Quadriceps Resistance Exercise on Functioning and Quality of Life in Patients with Knee Osteoarthritis: A Randomized Controlled Trial. Clin Rehabil, Vol. 30, 1074-1087.

World Health Organization, 2004, Appropriate Body-Mass Index for Asian populations and its Implications for Policy and Intervention Strategies. THE LANCET, Vol. 363, 157-163.
Yaser K. and Razie G., 2020, The Effect of a Water Jogging Exercise Course on Older Men with Knee Osteoarthritis. EQOL Journal, Vol. 12(2), 31-35. DOI:10.1007/s10120-011-0042-4.

Zampogna, B., Papalia, R., Papalia, G. F., Campi, S., Vasta, S., Vorini, F. and Denaro, V., 2020, The Role of Physical Activity as Conservative Treatment for Hip and Knee Osteoarthritis in Older People: A Systematic Review and MetaAnalysis. Journal of clinical medicine, Vol. 9(4), 1-20. doi:10.3390/jcm9041167. 